\title{
STRONG CP VIOLATION: THE NEUTRON ELECTRIC DIPOLE MOMENT IN LATTICE QCD*
}

\section{Andreas GOCKSCH}

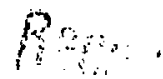

\section{Physics Department, Brookhaven National Laboratory, Upton, New York, 119ramn BNL -43520 \\ DE90 004952}

I report on an exploratory calculation of the neutron electric dipole moment induced by a $C P$ violating $\theta-\operatorname{term}$ in the QCD action within the framework of lattice QCD.

Some time ago it was discovered 1 that a term of the form (we use a euclidean metric throughout)

$$
\mathcal{L}_{\theta}=\frac{+i \theta g^{2}}{32 \pi^{2}} F F^{*}
$$

could be added to the QCD Lagrangian. Certainly one of the more dramatic consequences of having such a term present would be the nonvanishing of the neutron electric dipole moment (NEDM). This comes about because of the $P$ and $T$ violating nature of $\mathcal{L}_{\theta}^{*}$. Experimentally the situation is unclear at the moment. A russian experiment ${ }^{2}$ claims to find a non-vanishing dipole moment at the two sigma level: $d=-1.4(0.6) \cdot 10^{-25} e \cdot \mathrm{cm}$. On the other hand a european-american collaboration at the high flux reactor of the institut Laue-Langevin quotes a result consistent with zero ${ }^{3}: d=-3(4) \cdot 10^{-26} e \cdot \mathrm{cm}$. In any case the NEDM is certainly smaller than $O\left(10^{-25} e\right.$. $\mathrm{cm}$ ). With most theoretical analyses favoring a value of $d \sim 10^{-18} \theta \mathrm{e} \cdot \mathrm{cm}$ this leads to a bound on the vacuum angle of $\theta$ smaller than $O\left(10^{-\theta}\right)$. The smallness of $\theta$ is sometimes referred to as the strong $C P$ nroblem.

îhe above mentioned theoretical prediction for the NEDM comes about in the following way. The quantity we are after is

$$
\vec{d}=\left\langle N|\vec{d}| N>=Q \int d^{3} x<N\left|\vec{x} \bar{\psi} \gamma_{0} \psi(\vec{x})\right| N>.\right.
$$

It is non-vanishing only because of the explicit breaking of parity in the action due to the term in eq. (1). To compute the matrix element one employs a trick Because of the famous Adler-Bell-Jackiw Anomaly of the flavor singlet axial current $\mathcal{L}_{\theta}$ can be traded for a complex $\theta$ - dependent mass term by performing an axial rotation on the quark fields. Inserting a complete set of intermediate states in (2) thus leads to the computation of products of matrix elements of the form $m \theta<N|\vec{d}| X><X\left|\bar{\psi} i \gamma_{5} \psi\right| N>$ where $m$ is the current quark mass (I have assumed two degenerate light flavors and used the fact that $\theta$ is small to expand the exponential of the action). One than typically proceeds to decide on a particular set of states that one thinks are the important ones in the sum over $|X\rangle$ and computes the resulting matrix elements within a model that is supposed to capture the non-perturbative low energy dynamics of $Q C D$. Baluni ${ }^{4}$ uses the bag model, Crewther ${ }^{5}$ et. al. use an effective pion-nucleon lagrangian.

Clearly it would be desirable to compute (2) directly from QCD. Just as in the computation of weak interaction matrix elements ${ }^{6}$ lattice QCD is the ideal tool for performing such a calculation. Recently ${ }^{7}$ we have taken a first step in this direction. There are a number of difficulties one faces in such a project. In euclidean space $\mathcal{L}_{\theta}$ is imaginary and including (1) in the action (i.e. an appropriate lattice transcription of it) means having to do a complex action simulation, which at the present time is not possible. Alternatively, again expanding as above one obtains

$$
\vec{d}=\left\langle N\left|\vec{d} \cdot L_{\theta}\right| N\right\rangle
$$

But (3) is a very difficult quantity to measure on the lattice. It involves correlating the gluonic quantity $F F^{*}$ with all possible contractions of the quarks coming from the dipole operator and the nucleons.

"Talk presented at the Europhysics conference on High Energy Physics, Madrid, September 6-13 1989 
- The sonfigurations and propagatora however are now. generated with the standard action. Instead of using directly the electric dipole operator one can also introduce an external alectric field. This way one only has to compute matrix elements of the form $\left\langle N\left|F F^{*}\right| N\right\rangle$. The nucleon propagator now depends on the external field. But still one must correlate a lattice version of $F F^{*}$ with the nucleon propagator. This seems rather hard.

Fortunately the same trick that one uses in the continuum can be employed here. Utilizing the fact that the so called Wilson fermion method gives the correct anomaly of the singlet axial current on the lattice one can again use instead of (1) a term $M e^{i \text { s } \theta} \bar{\psi} \psi$ in the action $^{8}$. To extract the electric dipole moment of the nucleon we use the above mentioned external field technique which had previously been successfully applied to the computation of baryon magnetic moments on the lattice. ${ }^{9}$ In this background electric field we measure the difference between spin up and spin down masses. From it one can infer the value of the electric dipole moment at fixed $\theta$. We used the quenched approximation, i.e. the gauge configuration were generated using only the pure gauge action as a weight. This amounts to neglecting the effect of dynamical or sea quarks. To summarize: We computed neutron and proton spin up and down propagators that are made up of $\theta$ and $E$ (the external electric fieid) dependent quark propagators on quenched gauge configurations. This amounts to computing the matrix element $\left\langle N\left|M \theta \bar{\psi} i \gamma_{5} \psi\right| N\right\rangle$ in an external wield.

Without going into any of the technical details let me discuss our results. The value for the ratio of the neutron to proton EDM of $R=-1.7(.6)$ at a large value of the quark mass is close to what one would expect on the basis of a nonrelativistic $S U(6)$ quark model which predicts $R=-\frac{3}{2}$. This is interesting. It suggests that the bulk of the electric dipole moment of the nucleon in our calculation is just the sum of the quark electric dipole moments. Our estimate for the NEDM when converted into physical units using the value for the lattice spacing from DeGrand's ${ }^{10}$ work is $d \approx-4 \cdot 10^{-14} \theta e \cdot \mathrm{cm}$ with a (statistical) error of $1.4 \cdot 10^{-14} \theta \mathrm{e} \cdot \mathrm{cm}$. Taken at face value this translates into a bound of $\theta$ smaller than $O\left(10^{-11}\right)$. The magnitude of the dipole moment is about one hundred times larger than most continuum estimates of this quantity. Clearly our calculation is to a large extent an exploratory one. It must be repeated us- ing larger laritices, weaker couplings etc. But thare is one big difference between our calculation and the continuum ones which in our mind is to a large extent responsible for the big difference between the two. In the continuum one builds in chiral symmetry from the beginning. As a consequence the NEDM vanishes with the current quark mass which is the mass that enters the famous relation $m_{\pi} \sim \sqrt{m}$. Hence in the continuum we have $d=O\left(\frac{m_{n}^{2}}{M_{N}}\right)$. In our lattice calculation the NEDM vanishes with the bare lagrangian mass which is not the mass entering the current algebra relations (This is because Wilson fermions break the chiral symmetry, so there are mass counterterms which are not proportional to the bare mass). Hence on the lattice $d=O\left(\frac{1}{M_{N}}\right)$.

The fact that in our calculation the electric dipole moment of the neutron does not vanish with the pion mass (i.e. at $\kappa_{c}$ ) sounds alarming. One possibility is that this is indeed the wrong behavior and one needs to fix things up by coupling $e^{i \text { sio }}$ to the renormalized mass rather than the bare one ${ }^{11}$. On the other hand our result might be just be an indication of how careful one must be when dealing with an anomalous current - in particular non-perturbatively. It is a well known theorem that there exists no regularization scheme that respects both gauge and chiral symmetry. If one wants to get numibers out of the theory one must first regulate the theory. On the lattice, which is the only good scheme I know of that allows us to define the theory non-perturbatively, one breaks the chiral symmetry explicitly in order to get the correct anomaly in the axial current. This leads to mass counter terms and the loss of the naive relation between the bare lagrangian mass and the pion mass. The situation is entirely analogous to what one finds with a Pauli-Villars regulator in the continuum. To conclude, before one goes ahead and performs a similar calculation using bigger lattices and different parameters to come up with a real "lattice prediction" for the neutron electric dipole moment these theoretical issues have to be properly understood.

\section{ACKNOWLEDGEMENTS}

I recall with pleasure conversations with $\mathrm{G}$. Martinelli. This manuscript has been authored under Contract No. DE-AC02-76.CH00016 with the U.S. Department of Energy. The computations reported here were performed on a CRAY-2 computer at MFE. 


\section{REEERENCES}

1. G. 't Hooft, Phys. Rev. Lett. 37 (1983) 8

2. I.E. Altarev et. al., JETP Lett. 44 (1986) 460

3. D. Tompson, Talk presented at the Europhysics conference on High Energy Physics, Madrid, September 6-13 1989.

4. V. Baluni, Phys. Rev. D19 (1979) 2227

5. R.J. Crewther, P. Di Vechia, G. Veneziano and E. Witten, Phys. Lett. 88B (1979) 2227

6. F. Rapuano, these proceedings
-7. S. Aoki and A. Gocksch, Phys. Rev. Lett. 63 (1989) 1125

8. E. Seiler and I.O. Stamatescou, Phys. Rev. D25 (1982) 2177

9. C. Bernard, T. Draper, K. Olynik and M. Rushton, Phys. Rev. Lett. 49 (1982) 1076;

G. Martinelli, G. Parisi, R. Petronzio and F. Rapuano, Phys. Lett. 116B (1982) 434

10. R.D. Loft and T. DeGrand, Phys. Rev. D39 (1989) 2678

11. J. Smit, Acta Phys. Pol. B17 (1986) 531;

G. Martinelli, these proceedings

\section{DISCLAIMER}

This report was prepared as an account of work sponsored by an agency of the United States Government. Neither the United States Government nor any agency thereof, nor any of their employees, makes any warranty, express or implied, or assumes any legal liability or responsibility for the accuracy, completeness, or usefulness of any information, apparatus, product, or process disclosed, or represents that its use would not infringe privately owned rights. Reference herein to any specific commercial product, process, or service by trade namie, trademark, manufacturer, or otherwise does not necessarily constitute or imply its endorsement, recommendation, or favoring by the United States Government or any agency thereof. The views and opinions of authors expressed herein do not necessarily state or reflect those of the United States Government or any agency thereof. 